\title{
Dialogar con los alfareros e interpretar la cerámica precolonial andina
}

\section{Gabriel Ramón y Catherine Lara}

\section{Q OpenEdition \\ 1 Journals}

\section{Edición electrónica}

URL: https://journals.openedition.org/bifea/11498

DOI: $10.4000 /$ bifea.11498

ISSN: 2076-5827

\section{Editor}

Institut Français d'Études Andines

\section{Edición impresa}

Fecha de publicación: 1 abril 2020

Paginación: 1-18

ISSN: 0303-7495

\section{Referencia electrónica}

Gabriel Ramón y Catherine Lara, «Dialogar con los alfareros e interpretar la cerámica precolonial andina», Bulletin de l'Institut français d'études andines [En línea], 49 (1) | 2020, Publicado el 08 agosto 2020, consultado el 24 agosto 2021. URL: http://journals.openedition.org/bifea/11498 ; DOI: https:// doi.org/10.4000/bifea. 11498

\section{(c) (i) $\odot$}

Les contenus du Bulletin de l'Institut français d'études andines sont mis à disposition selon les termes de la licence Creative Commons Attribution - Pas d'Utilisation Commerciale - Pas de Modification 4.0 International. 


\title{
Dialogar con los alfareros e interpretar la cerámica precolonial andina*
}

\author{
Gabriel Ramón** \\ Catherine Lara***
}

\section{Introducción}

Los estudios reunidos en este volumen temático comparten un objetivo: discutir los usos de la etnografía alfarera en la interpretación del testimonio alfarero precolonial andino. Si bien se podría considerar una meta bastante específica, implica a la arqueología andina en conjunto. En primer lugar, sabemos que la cerámica es la evidencia más empleada en arqueología andina para narrar el pasado precolonial; por tanto, cualquier debate sobre interpretación alfarera repercutirá en la discusión sobre otros materiales (i.e. cestería, mates pirograbados, textiles...). En segundo lugar, se trata de un campo interdisciplinario que convoca permanentemente a la Etnografía y a la Arqueología, y permite cuestionar las relaciones entre ambas; es un campo que ha recibido múltiples denominaciones y ha permitido sofisticar las interpretaciones sobre la alfarería precolonial1.

Para discutir este tema, es preciso contar con plataformas académicas, sin embargo, por diversas razones, en Sudamérica hay pocos eventos que permitan

\footnotetext{
* Asumimos como «colonial» al período entre la invasión europea y el inicio de la época republicana.

** Profesor asociado, Departamento de Humanidades, Pontificia Universidad Católica del Perú (PUCP), Lima. E-mail: glramon@pucp.edu.pe

*** Investigadora principal, Instituto Francés de Estudios Andinos - IFEA (UMIFRE 17 MEAE / CNRS USR 3337 América Latina). E-mail: catherine.lara@cnrs.fr

1 Hay mucho debate sobre los distintos nombres de esta zona disciplinar. Algunos prefieren llamarla etnografía, otros etnoarqueología, otros arqueología a secas (dos referencias indispensables para
} 
intercambios entre arqueólogos de países andinos. Si bien desde hace algún tiempo varios de los autores del presente volumen nos conocíamos personal, virtual y/o bibliográficamente, desde 2016 aumentó el interés por formalizar esa interacción esporádica. El esperado diálogo se materializó en la IX Reunión de Teoría Arqueológica de América del Sur (TAAS, Ibarra, Ecuador, 2018), donde los editores organizamos una mesa de discusión sobre etnografía alfarera y Arqueología que contó con la participación de colegas de Argentina, Colombia, Ecuador y Perú. Debido al éxito de esta reunión, se organizó una segunda mesa en el marco del XVII Congreso de Antropología en Colombia (Cali, 2019) con colegas de los mismos países. Finalmente, pudimos incluir a un colega de Bolivia. Esta publicación es el fruto de todos esos diálogos previos que han permitido consolidar nuestro grupo de trabajo. No es casual que esta propuesta se haya materializado en el Boletín del Instituto Francés de Estudios Andinos, una institución dedicada a la integración de colegas de los países andinos.

El segundo objetivo de este número temático es contribuir a superar un problema de comunicación entre quienes trabajan en el campo de la etnografía alfarera orientada a la arqueología precolonial andina. Este aislamiento ha provocado que los autores andinistas, incluso de países vecinos, se ignoren entre ellos. Por otro lado, la ausencia de plataformas de discusión resulta en que los mencionados autores raramente citen fuentes no andinistas consideradas como referencias en la materia, especialmente aquellas no anglófonas. Consideramos que, como resultado de esta falta de interacción, la academia andinista prácticamente no tiene voz en el escenario mundial de la etnografía alfarera orientada a la Arqueología. Un ilustrativo ejemplo es Contesting Ethnoarchaeologies: Traditions, Theories, Prospects (Marciniak \& Yalman, 2013), que busca iluminar la producción no anglófona en este campo, y que, sin embargo, no incluye ningún autor de América Latina. En este contexto, el diálogo académico desde el sur, desde los Andes, se hace aún más necesario.

En el territorio andino (desde Argentina y Chile hasta Colombia), si bien la etnografía alfarera y la Arqueología pueden ser consideradas actividades independientes, hay un concepto que las ha asociado históricamente: la analogía etnográfica (Gándara, 1990; Perelman, 1997:153-166; Politis, 2015: 60; Williams, 2019: 45-48; Wylie, 1985). Más precisamente, se trata de lo que podríamos denominar el «pacto analógico andino». La analogía etnográfica puede definirse como la transmisión de información de un foro (presente etnográfico) a un tema (pasado precolonial), pero el «pacto analógico andino» implica algo más. Si, tomando en cuenta los límites de nuestra compilación, nos concentramos en la etnografía alfarera, el «pacto analógico andino» está claramente planteado en una observación de Rowe:

The more information we have on the modern pottery techniques in Peru, the better will we be able to interpret ancient ceramics (en O'Neale,1976: 41).

esta controversia son Gosselain, 2016 y Politis, 2015: 43). Este debate está lejos de culminar pero es dificil pensar que el camino sea cambiar una etiqueta y continuar con las mismas prácticas. El lector podrá notar que en este volumen temático cada autor tiene una perspectiva distinta respecto al término, diferencia que los editores hemos optado por mantener. 
Esta certera afirmación, en la que se podría reemplazar «Perú» por «Andes», contiene un principio base adoptado tácitamente por muchos investigadores: si el tema es precolonial andino, lo ideal es que el foro sea del presente etnográfico andino2. En eso consiste el «pacto analógico andino», que además abre toda una serie de posibilidades de discusión sobre la localización de foro y tema: ¿deben ser siempre de la misma región?, ¿es preferible que lo sean? ¿en qué criterios basarnos para responder a ello?3

¿Todos los arqueólogos andinistas usan la analogía etnográfica? La respuesta seguramente dividiría a los académicos, pero nosotros consideramos que incluso quienes no la reconocen como elemento fundamental de la interpretación arqueológica la emplean implícitamente. Esto remite al complejo tema de la relación histórica y conceptual entre Arqueología y Etnografía. Los andinistas normalmente emplean dos tipos de analogías etnográficas, la formal y la institucional (Ramón, 2008: 2-37). Aunque sus fronteras son difusas, la primera se vincula principalmente a la discusión de la función/uso de los objetos, y la segunda, a las formas de organización social. Así, por ejemplo, la primera discutiría si un artefacto encontrado en contexto precolonial sirvió para tejer o para hacer cerámica, asimismo se podría usar para reconstruir el proceso productivo a través de sus huellas. Mientras tanto la analogía institucional permitiría decidir si estamos ante la presencia de un taller alfarero organizado por grupos de familiares o no. La analogía formal es aplicada casi automáticamente y existe una metodología de trabajo clara; en el caso de la institucional, hay mayor debate respecto a los procedimientos analíticos (ver los diversos trabajos de este volumen).

Planteadas las líneas básicas de nuestra propuesta, conviene ahora explorar las relaciones entre etnografía alfarera y Arqueología desde una perspectiva histórica. Presentamos luego las tendencias y temas analíticos más recientes, para finalmente abordar el debate actual sobre la incorporación de las voces de los alfareros en la investigación.

\section{TENDENCIAS DIACRÓNICAS: ENTRE CASOS CONCRETOS Y GENERALIZACIÓN}

Al menos en Ecuador y Perú (países andinos donde, junto con Bolivia, se concentra la mayor cantidad de pueblos con alfareros rurales en actividad), las primeras etnografías alfareras se vinculan a la Arqueología, o son realizadas por personas interesadas por las «antigüedades»4. Podríamos dividir la producción

2 El «pacto analógico andino» no es direct historical approach; en todo caso es un requisito del mismo.

3 El caso de la arqueología caribeña o algunas tendencias ontológicas actuales, en las que muchas veces para buscar el foro etnográfico se acude a la experiencia continental sudamericana (amazónica), muestra que no siempre las regiones de foro y tema deben coincidir (ver adelante).

4 «Pueblos con alfareros rurales» alude sobre todo a los alfareros que trabajan en contextos rurales, con arcilla recogida en canteras no industriales y recurriendo a técnicas transmitidas por varias generaciones. 
posterior en dos grupos (no excluyentes, más bien en diálogo constante): las etnografías llevadas a cabo desde inquietudes antropológicas o sociológicas (es decir, esencialmente contemporáneas, ligadas muchas veces al significado actual de prácticas consideradas ancestrales), y aquellas realizadas por arqueólogos en el marco de problemáticas estrictamente precoloniales. Centrándonos en los casos de Ecuador y Perú, pero con una perspectiva andina global, evaluemos históricamente esa actividad interdisciplinaria5.

\section{1. De la exploración a las primeras síntesis}

En Perú, el primer reporte sobre alfareros de la costa o sierra es de Brüning (1898). Realizado en 1889, está dedicado a un alfarero itinerante que producía sus vasijas con la técnica del paleteado en la región de Olmos, al extremo sur del desierto de Sechura, en el departamento de Lambayeque. Entre 1908 y 1909, Barrett (1925: 173-181) realiza trabajo de campo con los cayapas (estribaciones occidentales del norte del Ecuador), e incluye una detallada descripción ilustrada de la cadena operativa alfarera. Esta investigación surge a raíz de una pregunta analógica en torno a los descubrimientos arqueológicos de Saville en el sitio de La Tolita (costa norte, relativamente cercano al territorio cayapa). De esta época datan los trabajos de Boman (1908: 478) sobre Argentina. Volviendo a Perú, en 1915, un ingeniero de minas suizo hizo un detallado registro de los alfareros pastores de Huayllay, departamento de Cerro de Pasco (Godet, 1918).

Luego de estos trabajos fundacionales, durante la primera mitad del siglo veinte, hubo dos grandes tendencias: los estudios sobre pueblos alfareros específicos y las generalizaciones. La primera consiste en reportes sobre pueblos con alfareros que aparecen en los más diversos formatos y detallan distintos aspectos de la actividad alfarera, principalmente manufactura y ocasionalmente distribución y uso. Por ejemplo, en Perú, la nota periodística de Espejo (1951) sobre Mangallpa, Cajamarca, o artículos como el de Tschopik (1950) sobre Chucuito, Puno, Christensen (1955) y Respaldiza (1953) sobre Simbilá, Piura, Collier (1959) sobre Mórrope, Lambayeque. En Colombia, Reichel-Dolmatoff (1945) sobre los Chamí y en Argentina Carman (1963) sobre Mina Clavero, Córdoba. Asimismo, las monografías que incluyen una sección sobre alfarería como la de Parsons sobre Otavalo, Ecuador (1945: 24, 25, láminas VIII, IX, X) y la de Stein sobre Hualcán, Ancash, Perú (1961: 91-95). Esta segunda etapa podría cerrarse con el valioso artículo de la arqueóloga Lavallée (1967) sobre Aco, Junín, Perú.

Paralelamente, desde muy temprano, también hubo un impulso generalizador. En este sentido, destaca el trabajo sobre las comunidades indígenas del sociólogo piurano Castro Pozo (1924), con información dispersa sobre 44 pueblos con

5 Nuestra discusión se limita al territorio andino, es decir las regiones conocidas como costa y sierra, excluyendo la Amazonía. Respecto a la bibliografía, por razones de espacio, solo escogemos algunos ejemplos que consideramos representativos. Para un panorama complementario, ver Sillar \& Ramón (2016). 
alfareros. Si bien no plantea una propuesta de conjunto ni aborda las técnicas de manufactura, deja en claro que la actividad alfarera está íntimamente vinculada con las comunidades andinas. El libro del arqueólogo sueco Linné (1925) es la primera síntesis específicamente dedicada a alfarería sudamericana, compilando todo lo conocido sobre etnografía alfarera para afinar la interpretación de la cerámica precolonial. No se trataba de un interés aislado: Linné (1925: 81) menciona un mapa de técnicas alfareras previamente elaborado por Uhle, el iniciador de la arqueología estratigráfica en los Andes.

Por la misma senda va el trabajo de Tello (1938), quien, tras comparar las técnicas de tres pueblos con alfareros (de Ancash, Cuzco y Lima), sostiene haber identificado la «técnica andina» que le permitirá interpretar la cerámica moche. Si bien posteriores investigaciones han mostrado que Tello ignoró la variabilidad técnica intra-andina (y simplificó la evidencia etnográfica), el objetivo de sistematizar la información para hacer analogías con material precolonial ha perdurado. Estas dos tempranas generalizaciones etnográficas elaboradas por arqueólogos confirman el estrecho vínculo entre ambas disciplinas, y el afán de construir un marco de referencia para las interpretaciones sobre cerámica precolonial. Finalmente, a medio camino entre las generalizaciones previas y la etapa siguiente, el antropólogo y novelista Arguedas (1989 [1940]) ensayó una rápida generalización sobre «cerámica popular andina».

\section{2. Folklorismo, indigenismo y cultura popular}

A partir de la década de 1960, los movimientos indigenistas/folkloristas y su institucionalización fomentaron la multiplicación de las etnografías alfareras en Ecuador y Perú. En Ecuador, el movimiento del folklorismo conoce su auge en esa década, en torno a la figura del antropólogo brasileño Carvalho Neto y de personalidades del mundo de las artes y letras como Carrión, Adoum, Guayasamín, entre otros. Este movimiento apuntaba al «conocimiento de nuestras raíces profundas, del trasfondo no explorado de la vida y conducta del hombre ecuatoriano» (Carrión, 1964: 23), cuyas costumbres y tradiciones heredadas a la vez de lo precolonial y de lo hispano se va a buscar documentar de forma sistemática y metódica (Carvalho Neto, ed., 1964: 68). Paralelamente, según las propias palabras de Carvalho Neto (ed., 1964: 66), se pretende denunciar la «explotación» del «indio oprimido». Se trata del auge del indigenismo; las leyes de reforma agraria verán la luz en 1964 y 1973 (García, 2011: 62). Tres etnografías alfareras aparecen en este contexto, sobre la Sierra Centro-norte del Ecuador (Tejada, 1966: 102) y la Sierra sur (Malo de Ramírez, 1975a; 1975b).

En Perú, la reforma agraria se realizó en la primera fase del Gobierno Revolucionario de las Fuerzas Armadas (GRFA) de Velasco (1968-1975). Si bien los reportes del Instituto Indigenista Peruano ya incluían información sobre alfarería (Arquinio et al., 1966; Fonseca et al., 1967), los estudios en este ámbito se multiplicaron durante el GRFA, que impulsó la documentación de la artesanía y la exhibición de cultura popular en los museos nacionales. Es así como, entre 1973 y 1974, 
el Museo de Arte e Historia de la Universidad Nacional Mayor de San Marcos auspició a Celia Bustamante y su equipo para estudiar alfareros en Cajamarca y Puno. Esta coyuntura favoreció asimismo las investigaciones de figuras significativas de la etnografía alfarera, quienes desarrollaron la dimensión regional de este tipo de estudio. La primera es Sabogal quien, junto a su equipo, produjo un extenso reporte sobre artesanía para el gobierno (Sabogal et al., 1974-1975), así como múltiples estudios regionales y una temprana tipología sobre la organización de la producción alfarera y los modos de distribución (Sabogal, 1978; 1982). Sabogal avanzó en ambas direcciones, tanto en el estudio de determinados pueblos con alfareros, como en la generalización sistemática. Aunque con un trabajo más discreto, cabe mencionar al etnógrafo suizo Spahni (1966), quien registró técnicas en pueblos con alfareros en diversas regiones de Perú (Ayacucho, Loreto, Piura, Puno). Otra figura emblemática de esta coyuntura es el arqueólogo Ravines, quien además de hacer etnografías alfareras específicas (1963-1964; 1966), elaboró un cuadro con 60 pueblos con alfareros de los Andes peruanos, prestando atención a variables como el género y las técnicas de manufactura (1971). Estas y otras temáticas serían retomadas por el arqueólogo estadounidense Kolb (1984) para discutir la vinculación entre producción alfarera y organización socioeconómica.

El GRFA coincidió asimismo con la llegada de investigadores polacos que produjeron una detallada etnografía de Caulimalca, sierra de La Libertad (Krzanowska \& Krzanowski, 1976). En paralelo, Krzanowska (1983) publicó el trabajo etnográfico comparativo más completo hasta entonces sobre alfarería en los Andes peruanos, que incluía un mapa y un detallado cuadro comparativo con 63 pueblos con alfareros. En la década de 1980 aparecerán estudios monográficos más detallados sobre pueblos con alfareros como el de Quiroz (1981) sobre Santo Domingo de los Olleros, Huarochirí, Camino (1982) sobre Simbilá, Piura, y Mohr (1984) sobre Raqchi, Cuzco.

En Ecuador, la producción científica de etnografías alfareras entre 1970 y 2000 fue principalmente promocionada por una institución creada por la Organización de Estados Americanos en 1975: el Centro Interamericano de Artesanías y Artes Populares (CIDAP). El objetivo inicial de esta iniciativa era capacitar artesanos, pero también documentar, estudiar y difundir sus conocimientos (Chamorro, 2006: 148-149). Las investigaciones del CIDAP introducen un cambio de enfoque, con el paso de la noción de folklore (centrada en el componente étnico), a aquella de cultura popular, es decir, a «un todo estructurado, con un corpus que nos permite hablar de cultura bajo los lineamientos antropológicos del término» (Naranjo, 1996: 15-16). La antropóloga sueca Sjöman es una figura central de las etnografías alfareras producidas por el CIDAP.

Si bien sus publicaciones se enfocan esencialmente en la Sierra sur del Ecuador (por ejemplo, Sjöman, 1989), Sjöman recorrió el país durante tres años (19871990), visitando 30 pueblos con alfareros y elaborando un inventario nacional de las técnicas alfareras. El resultado fue Vasijas de barro, la cerámica popular en el Ecuador (Sjöman, 1992). Considerando su detalle y amplitud, el enfoque regional de este proyecto supera largamente los de Krzanowska (1983), Ravines (1971) y 
Sabogal $(1978 ; 1982)$ en el Perú. La obra de Sjöman (1992: 11) busca destacar la voz del alfarero: originalmente, su libro iba a llamarse Voces de la tierra. Este objetivo lo encontramos también en otra etnografía del CIDAP realizada por Álvarez \& Domínguez (1987) en las comunidades de Río Verde y Buena Fe (actual provincia de Santa Elena, costa sur del Ecuador). Posteriormente, apareció el inventario alfarero sobre Bolivia que incluye 28 pueblos con alfareros (Sapiencia de Zapata et al., 1997).

El proyecto de investigación y publicación Tomos de cultura popular del Ecuador es otro aporte emblemático del CIDAP (Naranjo, 1966). Se trató de realizar un inventario de la cultura popular del país mediante un protocolo sistemático implementado por un equipo de investigadores generalmente dirigido por el antropólogo Naranjo. Estas investigaciones resultaron en 16 tomos publicados entre 1989 y 2010. Entre ellos, once incluyen recuentos sobre técnicas alfareras locales. Al margen del CIDAP, citaremos finalmente las etnografías alfareras realizadas por Lamas (1985: 80) en la Sierra norte del Ecuador, y Kennedy (1988) en la Sierra sur.

\section{ETNOGRAFÍA ALFARERA Y ARQUEOLOGÍA: TENDENCIAS METODOLÓGICAS Y TEMÁTICAS RECIENTES}

Como hemos podido observar, los arqueólogos en los Andes nunca han dejado de hacer etnografías alfareras o de emplearlas (Ramón \& Bell, 2013; Sillar \& Ramón, 2016). Sin embargo, desde los años 1980, es posible identificar una presencia sistemática de arqueólogos en esta actividad. Estos estudios se pueden vincular parcialmente al impacto de los estudios etnográficos llevados a cabo por la New Archaeology y las arqueologías posprocesuales (para un recuento del desarrollo de la etnoarqueología, ver Politis, 2015: 46-53; Williams, 2019: 59-84). La revisión panorámica de los trabajos correspondientes muestra que hay diferentes modos de combinar etnografía y arqueología. Existe asimismo una gran diversidad en las temáticas abordadas mediante este ejercicio. Si bien la frontera entre métodos y temáticas puede parecer tenue en ocasiones (los primeros estando a veces condicionados por las segundas), el presente apartado propone un recuento de las principales herramientas metodológicas y de las temáticas recientes más recurrentes en el uso arqueológico de la etnografía alfarera en los Andes.

Respecto a las herramientas metodológicas, cabe recordar que el trabajo etnográfico alfarero se apoya principalmente en tres tipos básicos de información interrelacionados: la materialidad (herramientas, materias primas, etc.), los gestos de los alfareros (acciones y posición del cuerpo mientras producen sus vasijas) y la oralidad (las explicaciones de los alfareros). Aquí nos enfocaremos en los métodos desarrollados en torno al estudio de la materialidad. Los dos últimos ámbitos (vinculados de forma más amplia a aquel del discurso), serán tratados en el apartado siguiente. 


\section{1. Tendencias}

De manera general, las etnografías interesadas en aspectos materiales buscan definir los indicadores empíricos característicos de fenómenos específicos. Por analogía, la presencia de los mismos indicadores en cultura material precolonial debe permitir sustentar su vinculación con el fenómeno en cuestión. Un ejemplo es la interpretación de Tschauner $(2009: 269$, 276) de los talleres cerámicos chimú de Pampa de Burros a partir de las inferencias etnográficas propuestas por Arnold (1999) en torno a técnicas y espacios físicos considerados como propios de una producción estandarizada. Más allá de la identificación general de objetos dentro de contextos espaciales específicos, los estudios sobre materialidad también pueden acudir a la arqueometría o conjunto de herramientas tomadas de las ciencias naturales para caracterizar materiales arqueológicos a nivel cualitativo y cuantitativo. Estos correlatos materiales son generalmente definidos según dos perspectivas distintas (no necesariamente excluyentes): 1) profundizando por igual cada una de las etapas del proceso de fabricación y uso de la cerámica, o 2) centrándose principalmente en las materias primas. Veamos a continuación en qué consisten estas dos perspectivas y cuáles son sus orígenes.

\section{1. 1. Estudios enfocados en la totalidad del proceso productivo y de uso}

Las principales referencias citadas en este ámbito por los arqueólogos andinistas son los manuales anglófonos de Shepard (1956), Rye (1981), Rice (2015, última reedición), y los de Balfet et al. (1992) y, en menor medida, Roux (2019) en el lado francófono. El trabajo de los españoles García Rosselló \& Calvo Trías (2013) es otro aporte significativo. El denominador común de estos manuales es la propuesta de protocolos de rastreo de técnicas y herramientas a partir de las huellas visibles en material arqueológico, definidos con base en análisis de laboratorio, estudios etnográficos y también experimentales.

Los manuales anglófonos mencionados no reivindican explícitamente alguna vinculación a una escuela de pensamiento en particular. Sus autores más bien subrayan su intención de basarse en evidencia estrictamente empírica. Quizás exista un vínculo entre el desarrollo de este interés por el conjunto de técnicas involucradas en la producción cerámica y una constatación que comienza a difundirse al menos desde principios del siglo XX, según la cual las formas y los diseños ya no son suficientes para definir asociaciones culturales (ver Brazzero, este volumen; Reichel Dolmatoff, 1945: 430; Holm, 1961: 153-154). A su vez, los manuales francófonos citados provienen de otro contexto, directamente ligado al concepto de «cadena operativa», originalmente discutido en los trabajos etnológicos de Mauss (1947) sobre la dimensión social de los hechos técnicos. Leroi-Gourhan (1964) propone luego una de las primeras definiciones de «cadena operativa», la cual será posteriormente desarrollada y diversificada por el movimiento Techniques et Culture. Este último inspiró asimismo en parte el manual de García Rosselló \& Calvo Trías (2013: 5). 
Estos manuales (en especial anglófonos) y la noción de «cadena operativa» recibieron una amplia acogida en la arqueología andina. No obstante, la aplicación completa de sus protocolos de reconocimiento de huellas a estudios conjuntos de material arqueológico y etnográfico es aún escasa en la región (Carmichael, 1986; Cleland \& Shimada, 1998; Gabelmann, 2014; Lara, 2017).

\section{1. 2. Estudios enfocados en materias primas y quema}

Este tipo de trabajo profundiza esencialmente los aspectos de la cadena operativa ligados a la procedencia y preparación de las materias primas (arcillas, desgrasantes y demás materiales usados en los tratamientos de superficie), y eventualmente la quema (Wagner et al., 1994). De manera general, estos estudios se apoyan principalmente en análisis físico-químicos, mineralógicos, petrográficos y composicionales (ver de la Fuente et al., este volumen). En Ecuador y Perú, los análisis petrográficos y químicos de las arcillas usadas en la fabricación de alfarería precolonial comenzaron a desarrollarse a partir de los años 1960 y 1970 (Ghezzi, 2011: 3, ver también Pereyra \& Puente, 2018: 6 para los casos de Argentina y Chile). Los trabajos de Arnold (1985) o Druc (por ejemplo Druc \& Velde, 2021) son actualmente considerados como referencias obligadas en este ámbito. Arnold asocia su propuesta a la ecología cultural, lo cual explicaría este interés marcado hacia las materias primas. Una de sus inferencias etnográficas más citadas por los arqueólogos es aquella del «umbral», o distancia promedio que suele observarse entre las fuentes de arcilla y los lugares de producción (Arnold, 1985: 35-57).

La popularización de este método dio pie al desarrollo de estudios etnográficos de talleres alfareros (en ocasiones ubicados cerca de sitios arqueológicos), para mejorar la lectura e interpretación de pastas precoloniales, y eventualmente ubicar posibles canteras de uso precolonial (Cadena, en este volumen; Cremonte, 1989; Druc et al., 2013; Minc et al., 2016; Serrano, en este volumen). Este tipo de referencias etnográficas es asimismo comúnmente citado en las investigaciones estrictamente basadas en análisis de laboratorio (Carosio et al., 2019: 358; Villanueva, en este volumen).

\section{2. Temáticas}

La arqueología andina suele acudir a la etnografía alfarera para esclarecer aspectos empíricos muy puntuales y concretos ligados a la cultura material, pero también para lidiar con ámbitos mucho más abstractos, como la organización del proceso productivo, la identidad (en un sentido amplio, es decir social, étnica, de género, generación, etc.) o las percepciones de los artesanos. Explorando este abanico de temáticas desde lo material hacia lo inmaterial, identificamos un primer grupo de trabajos enfocados en elementos muy concretos del proceso productivo. Aquí se encuentran por ejemplo las investigaciones sobre el papel desempeñado por los factores naturales (estacionalidad, altitud, entre otros) en las diferentes etapas de la fabricación de cerámica (Arnold, 1985; Cremonte, 1989: 119; Druc, 2001: 171). 
Otro ámbito donde la arqueología examina muy de cerca el dato etnográfico es el de la estructuración espacial y física de los talleres alfareros, potencialmente útil para la detección de espacios de producción precolonial (Llinás et al., 2019; Shimada, 1994: 295; Vargas, Ms, 2018: 1). Los aspectos tafonómicos, o relacionados con la función de las vasijas (García, 1988; Hayashida, 2009; Sillar, 2000), son asimismo recurrentes en la exploración etnográfica de los arqueólogos.

Siguiendo con campos más complejos e inmateriales del proceso productivo, están los estudios sobre organización y distribución de la producción, potencialmente reveladores de la estructura sociopolítica de los colectivos. Temáticas sobresalientes en este ámbito son aquellas de la organización doméstica y la división del trabajo (Cremonte, 1984; 1989; López, 2014; Sillar, 2000). En el ámbito de la variabilidad de modalidades de producción, la producción itinerante ha recibido mayor atención recientemente (Ramón, en este volumen). La especialización es asimismo un aspecto que ha interesado particularmente a los arqueólogos desde un punto de vista etnográfico (Valdez, 1997; De la Fuente et al., en este volumen), también en el marco del debate sobre la relación entre especialización y «complejidad social». La exploración de estos aspectos ha evidenciado la necesidad de adoptar perspectivas más amplias, como lo muestran las investigaciones centradas en la relación entre producción, distribución y demanda (Cremonte, 1984; 1989; López, 2014; Ramón \& Bell, 2013; Sillar, 2000). En este sentido, destacan los estudios del vínculo entre alfarería y otras actividades productivas (Cleland \& Shimada, 1998; Valdez ,1997).

El tema de la relación entre tecnología cerámica e identidad es otro campo dominante en el uso arqueológico de etnografías alfareras (Castro et al., 2017: 323; Gabelmann, 2014: 44; Mohr, 1984: 162; Monzón, 1991; Pozzi-Escot et al., 1993: 484; Ramón, 2008; Russell et al., 1994). Una problemática que sobresale en ese sentido es la ruptura o continuidad entre prácticas precoloniales y actuales (ver los estudios de Brazzero y Lara, en este volumen). También se ha trabajado sobre los préstamos técnicos, potencialmente útiles en la comprensión de los cambios visibles en el registro arqueológico (Roux \& Lara, 2016; Sillar, 1997).

\section{LAS VOCES DE LOS ALFAREROS: (DE)ONTOLOGÍAS Y OTRAS AGENDAS PENDIENTES}

Junto con la sofisticación técnica de los estudios alfareros tratada en la sección previa, hay otro asunto que va cobrando mayor importancia: la incorporación de las voces de los alfareros. Claramente impactados por el enorme prestigio del perspectivismo amazónico en la academia europea, ahora los arqueólogos andinistas no solo quieren registrar los datos sobre el «otro» sino también tomar en serio su perspectiva. No se trata de un ejercicio sencillo, ya que puede llegar a cuestionar el conjunto de la disciplina. En este sentido, la arqueología andina todavía está en una etapa de experimentación y desconcierto6. El debate sobre

6 Sobre el desconcierto, ver el informativo lapsus doble en el artículo introductorio del Boletín de Arqueología PUCP 24:12 (2018). Donde debían ir las referencias bibliográficas a la «arqueología 
el «giro ontológico» ha abierto una serie de posibilidades interpretativas en arqueología, directamente vinculadas con las discusiones de este volumen (ver los estudios de Ávila y Castellanos). Como bien indica Alberti (2016:175), en Arqueología, el reto es «trabajar con nuestro material de modo que maximice el potencial para que aparezca la diferencia ontológica».

Este reto provoca, al menos, tres cuestionamientos. Primero, la localización de las ontologías: ¿por qué el modelo animista o perspectivista amazónicos sería aplicable a la evidencia precolonial andina? ¿No sería más adecuado generar y aplicar modelos ontológicos locales? (ver las críticas de Bartolomé, 2015; Ramos, 2012). Segundo, la variabilidad intraandina: ¿̇hasta qué punto se puede aplicar conceptos identificados para Cuzco al resto de los Andes? Una manera de avanzar en este punto sería tomar en serio la literatura gris sobre los Andes, generalmente publicada en castellano. Tercero, ¿cómo medir la diferencia ontológica en el registro arqueológico? ¿Con los métodos occidentales, o debemos también introducir un giro ontológico en nuestra disciplina, más allá de incorporar algunos términos en aimara o quechua? Son temas puestos en agenda gracias a la saludable irrupción perspectivista, pero poco discutidos a nivel metodológico y material entre arqueólogos7.

Desde Brüning y Barrett, los investigadores han interactuado con los alfareros, aunque de forma distinta. Si revisamos los trabajos de los autores registrados en nuestro recorrido histórico, podemos observar una tendencia (con excepciones): mientras los antropólogos que hacían etnografía citan las voces de los alfareros, estas difícilmente aparecen en los trabajos arqueológicos (compárense Respaldiza, 1953; Camino, 1982 y Sjöman, 1992 con Arnold, 1985; Lavallée, 1967; Shimada, 1994). Los etnógrafos que consiguieron replantear su disciplina lo hicieron conversando con la gente y rescatando sus voces. En tal sentido, la etnografía alfarera ha sido siempre una plataforma ideal para percibir la diferencia ontológica (para ejemplos extraandinos ver Gallay, 1970; Gosselain, 1999). Esto nos conduce a una pregunta crucial: si los arqueólogos soslayamos las voces de los alfareros en etnografía, ¿podemos soñar con ontologías basadas en la cerámica precolonial? En un contexto donde las identidades de los emisores del discurso toman un papel crucial, es evidente que el «pacto analógico andino» debe incorporar el diálogo.

Reconocida la necesidad de incorporar las voces de los alfareros, surgen interrogantes sobre cómo hacerlo o incluso de la legitimidad de quienes pueden hacerlo. Aquí nos enfrentamos a un complejo dilema: en ciertos espacios académicos, es complicado divulgar los nombres de los alfareros con quienes se trabaja. Sin embargo, ise puede rescatar la voz del alfarero en textos que modifican el nombre del pueblo estudiado (Hosler, 1996), o en textos donde los alfareros

perspectivista» solo dice: «Referencia a la arqueología perspectivista». Lo mismo sucede con la «Referencia a la arqueología indígena/decolonial» [http://revistas.pucp.edu.pe/index.php/ boletindearqueologia/article/view/20751/20518, consultado 26.11.2020].

7 La introducción a la compilación de Lozada \& Tantaleán (2019) acierta en reconocer la potencial pluralidad de ontologías andinas, pero evita cualquier polémica, incluyendo los tres cuestionamientos indicados. 
son básicamente anónimos (Arnold, 1985)? Afortunadamente, los arqueólogos que hacen etnografía alfarera están cambiando sus estrategias e incorporando testimonios de sus entrevistados para cuestionar sus propias perspectivas. Sin embargo, no se trata de crear paraísos artificiales, la etnografía alfarera afronta retos deontológicos permanentes como el planteado por Matayoshi en su artículo sobre el pueblo con alfareros de Aco, Junín. Al concluir su texto, uno de sus entrevistados le increpa (nos increpa):

Usted te irás, pues. A tu casa te irás y te olvidarás de nosotros. Así estarás visitando todos los pueblos, para que te cuenten cómo vive el campesino y después se olvidará de nuestra pobreza. Así ha sido siempre, pero un día de estos, diferentes van a ser las cosas. Nosotros iremos a las ciudades, como perros andaremos olisqueando, entonces les haremos preguntas y apuntaremos en nuestros cuadernos. Así haremos, nos iremos a nuestras chacras y de ahí, leeremos lo que hemos escrito y nos olvidaremos de ustedes (Matayoshi, 1988:13).

Este incómodo discurso (entre ficcional y real) no le sonará ajeno a cualquiera que haya hecho trabajo de campo en los Andes como arqueólogo o etnógrafo. Hay un desafío político latente en nuestra disciplina (Ramón, 2013: 38-39).

Situados en este complicado escenario, es preciso considerar algunas interrogantes finales: ¿cuáles son los límites de pretender rescatar la voz del alfarero?, ¿cómo se vincula nuestro ejercicio etnográfico con el punto de vista indígena/local?, ¿qué cambia en nuestra interpretación?, ¿incorporamos realmente la voz del otro, o al final nos expresamos a través del otro? ¿cómo devolvemos el conocimiento recibido? Estas dudas deben ser parte explícita de las discusiones y servir para cuestionar los límites de nuestra disciplina (sin obviar las certeras críticas de Gosselain, 2016).

\section{CIERRE}

En teoría, si bien los modelos sobre alfareros precoloniales pueden producirse a partir de fuentes históricas, arqueológicas y/o etnográficas, en la práctica, este último tipo de evidencia ha sido el que ha guiado la lectura de los otros tipos de fuentes. La etnografía provee «visibilidad» tanto a la evidencia histórica como arqueológica. Como lo vimos al discutir las técnicas analíticas, y se podría afirmar también para los enfoques ontológicos, las preguntas y nuevos planteamientos que han sacudido buena parte de los lugares comunes en los que se apoyaban los estudios de laboratorio han surgido desde la etnografía. Aunque sea de manera indirecta y/o inconsciente, prácticamente todo arqueólogo recurre a la experiencia etnográfica. En ese sentido, lejos de ser un añadido o una «disciplina auxiliar», la etnografía alfarera ha sido históricamente constitutiva de la arqueología (cf. Gándara, 1990: 45-46, 76). Más allá de reconocer este vínculo, nuestra propuesta es ponerlo en práctica explícitamente en los siguientes artículos. 


\section{Agradecimientos}

GR. A María Patricia Ordoñez, la causante. A José Luis Pino y Jan Szemiński por compartir su saber. A Lupe Camino por guiar mi curiosidad. A la memoria de Irmild Wüst y su recordado curso en el MAE, USP. A todos los colegas y amigos que participaron en ambos eventos. A Alina Wong, que hubiera estado feliz con este volumen.

\section{Referencias citadas}

ALBERTI, B., 2016 - Archaeologies of Ontology. Annual review of anthropology, 45: 163179.

ÁLVAREZ, S. \& DOMÍNGUEZ, V., 1987 - La cerámica punteña actual. Revista del CIDAP 25: 93-112.

ARGUEDAS, J., 1989 [1940] - La cerámica popular india en el Perú. In: Indios, mestizos y señores: 60-65; Lima: Editorial Horizonte.

ARNOLD, D., 1985 - Ceramic Theory and Cultural Process, xi +268 pp.; Cambridge: Cambridge University Press.

ARNOLD, D., 1999 - Advantages and Disadvantages of Vertical Half-Molding Technology: Implications for Production Organization. In: Pottery and people (J. M. Skibo \& G. M. Feinman, eds.): 59-80; Salt Lake City: University of Utah Press.

ARQUINIO, J., BOLUARTE, F., FONSECA, C., GALDO, R., GHERSI, H., MARTÍNEZ, H., MURRUGARRA, J., \& TURPAUD, R., 1966 - Sociedad, cultura y economia en 10 areas andino-peruanas. Asilo, Chumabo, Chuyas-Huaychao, Huata y Quita, Julcamarca, Layo, Pirapi, Soras, Taraco, Yancao, sin paginado; Lima: Ministerio de Trabajo y Comunidades, Instituto Indigenista Peruano.

BALFET, H., FAUVET-BERTHELOT, M. F. \& MONZON, S., 1992 - Normas para la descripción de vasijas cerámicas, 146 pp.; México: CEMCA.

BANKES, G., 1988 - Paddle and anvil potters of the North Coast of Peru. In: Recent Studies in Pre-Columbian Archaeology (N. Saunders \& O. de Montmollin, eds.): 545-563; Oxford: B.A.R.

BARRETT, S., 1925 - The Cayapa Indians of Ecuador, Part I, 181 pp.; Nueva York: Museum of the American Indian, Heye Foundation.

BARTOLOMÉ, M., 2015 - El regreso de la barbarie. Una crítica etnográfica a las ontologías "premodernas". Trace, 67: 121-149.

BOMAN, E., 1908 - Antiquités de la région andine de la république Argentine et du désert d'Atacama, 2 vols.; xi + 948 pp.; París: Impr. Nationale, Librairie H. Le Soudier.

BRÜNING, H., 1898 - Moderne Töpferei der Indianer Perus. Globus. Illustrierte Zeitschrift für Länder- und Völkerkunde, 74: 259-260.

CAMINO, L., 1982 - Los que vencieron al tiempo. Simbilá, Costa Norte. Perfil etnográfico de un centro alfarero, 137 pp.; Piura: Centro de Investigación y Promoción del Campesinado.

CARMAN, M., 1963 - Una comunidad de alfareros. Cuadernos del Instituto Nacional de Antropología, 4: 35-66.

CARMICHAEL, P., 1986 - Nasca Pottery Construction. Nawpa Pacha, 24 (1): 31-48.

CAROSIO, S. A., SABATINI, G. \& CAHIZA, P.-A., 2019 - Prácticas de manufactura alfarera de las comunidades aldeanas de inicios del primer milenio (siglos III-VI DC) en el 
Noroeste argentino. Estudios de pastas cerámicas de Uchuquita (Anillaco, La Rioja). Chungara, 17 (3): 339-362.

CARRIÓN, B., 1964 - Carvalho Neto en la casa de la cultura ecuatoriana. In: Diccionario del Folklore Ecuatoriano (P. Carvalho-Neto, ed.): 21-26; Quito: Editorial de la CCE.

CARVALHO-NETO, P. (ed.), 1964 - Diccionario del Folklore Ecuatoriano, 483 pp.; Quito: Editorial de la CCE.

CASTRO PRIEGO, M., LABRADA OCHOA, M. O. \& CHASING GUAGUA, E. A., 2017 Mujer y tradición cerámica en la costa ecuatoriana: indicadores etnoarqueológicos. In: Museos arqueológicos y género. Educando en igualdad (L. Prados Torreira \& C. López Ruiz, eds.): 323-357/; Madrid: Ediciones Universidad Autónoma de Madrid.

CASTRO POZO, H., 1924 - Nuestra Comunidad Indígena, xxiv + 498 pp.; Lima: El Lucero.

CHAMORRO, I. G., 2006 - Artesanías y Cooperación en América Latina y el Caribe: Programa de la OEA (1969-1989), 256 pp.; Cuenca: CIDAP.

CHRISTENSEN, R., 1955 - Una industria moderna de cerámica en Simbilá, cerca de Piura, Perú. Chimor, Boletín del Museo de Arqueología de la Universidad Nacional de Trujillo, 3 (1): 10-20 + 6 figs.

CLELAND, K. \& SHIMADA, I., 1998 - Paleteada Potters: Technology, Production Sphere, and Sub-Culture in Ancient Peru. In: Andean Ceramics: Technology, Organization and Approaches (I. Shimada, ed.): 111-150; Philadelphia: University of Pennsylvania Museum of Archaeology and Anthropology.

COLLIER, D., 1967 - Pottery Stamping and Molding on the North Coast of Peru. In: Peruvian Archaeology: Selected Readings (J. Rowe \& D. Menzel, eds.): 264-274; Palo Alto: Peek Publications.

CREMONTE, M. B., 1984 - Alfareros itinerantes de los Colorados (Dto. Tafi, Tucuman). Aproximaciones a un estudio de etnografía arqueológica. Runa. Archivos para las Ciencias del Hombre, XIV: 247-263.

CREMONTE, M. B., 1989 - La alfarería tradicional actual: reflexiones y posibles aplicaciones para la Arqueología a través de dos casos de estudio. Runa, XIX (1): 117-133.

DRUC, I., 2001 - ¿Shashal o no shashal? Esa es la cuestión. Etnoarqueología cerámica en la zona de Huari, Ancash. Bulletin de l'Institut Français d'Études Andines, 30 (1):157173.

DRUC, I., INOCUCHI, K. \& ZHIZHANG, S., 2013 - Análisis de arcillas y material comparativo por medio de difracción de rayos $X$ y petrografía para Kuntur Wasi, Cajamarca, Perú. Arqueología y Sociedad, 26: 91-109.

DRUC, I. \& VELDE, B.,-2021 - Ceramic Materials in Archaeology, 310 pp.; Blue Mounds: Deep Education Press.

ESPEJO, J., 1951 - Los alfareros de Manka-Allpa. El Comercio, 1 diciembre: 3, 5.

FONSECA, C., ARQUINIO, J. \& GAMARRA, A. M., 1967 - Situación actual de las comunidades de Catac, Mita, Huata y Tumpa: Callejón de Huaylas, Ancash, 2 vols.; Lima: Instituto Indigenista Peruano.

GABELMANN, O. U., 2014 - Diversas preferencias tecnológicas: reconstruyendo la cadena operatoria de la producción cerámica del período Formativo en Cochabamba, Bolivia. In: Rebelión de los objetos: enfoque cerámico. Anales de la reunión anual de etnologia (E. Espejo \& M. Eyzaguirre, eds.): 39-63; La Paz: Musef.

GALLAY, A., 1970 - La poterie en pays Sarakolé (Mali, Afrique Occidentale). Journal de la Société des Africanistes, 40 (1): 7-84.

GÁNDARA, M., 1990 - La analogía etnográfica como heurística: lógica muestreal, dominios ontológicos e historicidad. In: Etnoarqueología. Primer Coloquio Bosch-Gimpera (Y. Sugiura \& M. C. Serra, eds.): 43-82; México D.F.: Universidad Autónoma de México. 
GARCÍA, L., 1988 - Etnoarqueología: manufactura de cerámica en Alto Sapagua. In: Arqueología Contemporánea Argentina. Actualidad y perspectiva (H. Yacobaccio, L. A. Borrero, L. C. García, G. G. Politis, C. A. Aschero, C. Bellelli, eds.): 33-58; Buenos Aires: Búsqueda.

GARCÍA S., F., 2011 - La construcción del pensamiento antropológico ecuatoriano: derroteros y perspectivas. Alteridades, 21 (41): 61-68.

GARCÍA ROSSELLÓ, J. \& CALVO TRÍAS, M., 2013 - Making pots: el modelado de la cerámica y su potencial interpretativo, 497 pp.; Oxford: British Archaeological Reports.

GHEZZI, I., 2011 - El análisis composicional en el estudio de la producción y distribución de la cerámica prehispánica, Bulletin de l'Institut français d'études andines, 40 (1): 1-29.

GODET, E., 1918 - Monographie de la région de Huancavelica (Département de Junín) Pérou. Bulletin de la Société Neuchateloise de Géographie, XXVII:121-182.

GOSSELAIN, O. P., 1999 - In Pots we Trust: The Processing of Clay and Symbols In SubSaharan Africa. Journal of Material Culture, 4 (2): 205-230.

GOSSELAIN, O. P., 2016 - To hell with ethnoarchaeology! Archaeological Dialogues, 23 (2): 215-228.

HAYASHIDA, F., 2009 - Chicha Histories: Pre-Hispanic Brewing in the Andes and the Use of Ethnographic and Historical Analogues. In: Drink, Power, and Society in the Andes (J. Jennings \& B. Bowser, eds.): 232-256; Gainsville: University Press of Florida.

HOLM, O., 1961 - La técnica alfarera de Jatunpamba (Ecuador). Cuadernos de Historia y Arqueología, X (27): 153-210 + 4 láminas.

HOSLER, D., 1996 - Technical Choices, Social Categories and Meaning Among the Andean Potters of las Ánimas. Journal of material culture, 1 (1): 63-92.

KENNEDY-TROYA, A., 1988 - Pompilio y su pueblo. Exposición de cerámica de Chordeleg, 50 pp.; Cuenca: Fundación Paul Rivet.

KOLB, C. C., 1984 - The Methodology of Latin American Ceramic Ecology, 39 pp.; Colorado: Museum of Anthropology, University of Northern Colorado. Museum of Anthropology Miscellaneous Series.

KRZANOWSKA, R., 1983 - Peruwiańska ceramika ludowa. Etnografia Polska, 27: 173-201.

KRZANOWSKA, R. \& KRZANOWSKI, A., 1976 - Garncarstwo ludowe w dolinie Alto Chicama w północnym Peru. Etnografia Polska, 20: 185-214.

LAMAS D., V., 1985 - La alfarería tradicional utilitaria en el área de Otavalo y sus inmediaciones". Sarance 10: 79-126.

LARA, C., 2017 - Aportes del enfoque tecnológico a la arqueología precolombina: pasado y presente de la alfarería en el valle del río Cuyes y su región (Andes sur-orientales del Ecuador), 240 pp.; Oxford: Archaeopress.

LAVAlLÉE, D., 1967 - La poterie de Aco (Andes centrales du Pérou). Objets et Mondes, VII (2): 103-120.

LEROI-GOURHAN, A., 1964 - Le geste et la parole, tome 1, Technique et langage, 325 pp.; París: Albin Michel.

LINNÉ, S., 1925 - The Technique of South American Ceramics, 199 pp.; Gotemburgo: Elanders Boktryckeri Aktiebolag.

LLINÁS ZAMBRANO, J., NOVILLO VERDUGO, M. \& ORTIZ NARANJO, J., 2019 - Análisis comparativo de talleres alfareros México-Ecuador: una mirada etnoarqueológica. Temas Antropológicos, Revista Científica de Investigaciones Regionales 41 (2): 61-78. 
LÓPEZ, M., 2014 - Casira y la Manka fiesta, Jujuy, Argentina. Observaciones actualísticas sobre la producción de piezas cerámicas para el intercambio e implicancias en la distribución de piezas arqueológicas en la Quebrada de Humahuaca. In: Rebelión de los objetos: enfoque cerámico (E. Espejo \& M. Eyzaguirre, eds.): 201-222; La Paz: Musef.

LOZADA, M. \& TANTALEÁN, H. (eds.), 2019 - Andean ontologies. New Archaeological Perspectives, 384 pp.; Gainsville: University Press of Florida.

MALO DE RAMÍREZ, G., 1975a - Alfarería y orfebrería de Chordeleg. In: Recopilación de investigaciones sobre artesanía (Instituto Azuayo de Folklore, ed.): 11-35; Cuenca: Editorial de la CCE.

MALO DE RAMÍREZ, G., 1975b - Alfarería: las ollas de barro de San Miguel de Porotos. In: Recopilación de investigaciones sobre artesanía (Instituto Azuayo de Folklore ed.): 36-48; Cuenca: Editorial de la CCE. MARCINIACK, A. \& YALMAN, N., 2013 - Contesting Ethnoarchaeologies, Traditions, Theories, Prospects, 264 pp.; Nueva York: Springer Verlag.

MATAYOSHI, N., 1988 - Mancalluta. Relatos orales de ceramistas. Kamaq Maki, 2:12-13.

MAUSS, M., 1947 - Manuel d'ethnographie, 211 pp.; París: Payot.

MINC, L., YANCHAR, K., BRAY, T. \& ECHEVERRÍA ALMEIDA, J., 2016 - Potting Clays and Ceramic Provenance in Northern Highland Ecuador. In: Vessels Explored: Applying Archaeometry to South American Ceramics and their Production (E. M. Stovel \& G. A. de la Fuente, eds.): 47-63; Oxford: BAR.

MOHR CHÁVEZ, K. L., 1984 - Traditional pottery of Raqch'i, Cuzco, Peru: a preliminary study of its production, distribution and consumption. Ñawpa Pacha, 22-23: 161 210.

MONZON, S., 1991 - El estudio de la cerámica y su contribución a una investigación interregional - el caso de Piura. Bulletin de I'Institut Français d'Études Andines, 20 (2): 589-597

NARANJO, M., 1996 - La cultura popular en el Ecuador: Cotopaxi, 226 pp.; CIDAP: Cuenca. Colección "La cultura popular en el Ecuador", t. II.

O'NEALE, L. M., 1976 - Notes on pottery making in Highland Peru. Nawpa Pacha, 14 (1): 41-59.

PARSONS, E. C., 1945 - Peguche, Canton of Otavalo, Imbabura, Ecuador: A Study of Andean Indians, 225 pp.; Chicago: University of Chicago Press.

PERELMAN, C., 1997 - El imperio retórico: retórica y argumentación, 214 pp.; Bogotá: Norma.

PEREYRA DOMINGORENA, L. \& PUENTE, V., 2018 - Presentación. Comechingonia, 22 (1): 5-9.

POLITIS, G. G., 2015 - Reflections on Contemporary Ethnoarchaeology. Pyrenae, 46 (1): 41-83.

POZZI-ESCOT, D., ALARCÓN, M. M., VIVANCO P., C., 1993 - Instrumentos de alfareros de la época wari. Bulletin de I'Institut Français d'Études Andines, 22 (2): 467-496

QUIROZ, G., 1981 - La tradición alfarera en Santo Domingo de los Olleros, 93 pp.; Lima: Seminario de Historia Rura Andina.

RAMÓN, G., 2008 - Potters of the Northern Peruvian Andes: a palimpsest of technical styles in motion, xix + 437 pp.; Norwich: Universidad de East Anglia. Tesis doctoral.

RAMÓN, G., 2013 - Los alfareros golondrinos. Productores itinerantes de los Andes, 167 pp.; Lima: IFEA, Sequilao Editores. 
RAMÓN, G. \& BELL, M., 2013 - Re-placing plainware: Production and distribution of domestic pottery, and the narration of the pre-colonial past in the Peruvian Andes. Journal of Anthropological Archaeology, 32 (4): 595-613.

RAMOS, A. R., 2012 - The Politics of Perspectivism. Annual Review of Anthropology 41: 481-494.

RAVINES, R., 1963-1964 - Alfarería doméstica de Huaylacucho, Departamento de Huancavelica. Folklore Americano 11-12:92-96.

RAVINES, R., 1966 - Caccasiri-pi rurani mankata. Folklore Americano 14: 210-222.

RAVINES, R., 1971 - La alfarería tardía en el valle del Mantaro: una aproximación ecológica, 194 pp.; Lima: Universidad Nacional Mayor de San Marcos. Tesis de bachillerato.

REICHEL-DOLMATOFF, G., 1945 - La manufactura de cerámica entre los Chamí. Boletín de Arqueología, 1 (1): 425-430.

RESPALDIZA, J., 1953 [1945] - Un mito y un cuento de Simbilá. Folklore Americano, 1: 82-100.

RICE, P., 2015 - Pottery analysis, a sourcebook, 592 pp.; Chicago: University of Chicago Press.

ROUX, V., 2019 - Ceramics and Society: a Technological Approach to Archaeological Assemblages, 329 pp.; Cham: Springer.

ROUX, V. \& LARA, C., 2016 - Why potters have not borrowed the kiln? Comparing narratives of Indian and Ecuadorian potters. Americae [en línea], 1: 21-35.

RUSSELL, G. S., BANKS, L. L. \& BRICEÑO ROSARIO, J., 1994 - Cerro Mayal: nuevos datos sobre la producción de cerámica Moche en el valle de Chicama. In: Moche, propuestas y perspectivas (S. Uceda \& E. Mujica, eds.): 181-206; Lima: IFEA, Universidad Nacional de La Libertad-Trujillo.

RYE, O. S., 1981 - Pottery Technology. Principles and Reconstruction, 150 pp.; Washington: Taraxacum.

SABOGAL, J., 1978 - Siete sitios cerámicos en la región de Piura, 47 pp.; Lima: Ministerio de Trabajo/ Ministerio de Industria.

SABOGAL, J., 1982 - La cerámica de Piura, 2 vol.; Quito: IADAP.

SABOGAL, J., ÁlVAREZ, T., ESCOBAR, A., VILDOSOLA, J. \& MORANTE, M., 1974-1975 - Estudio Socio-Económico del ámbito cultural, 4 vols.; Lima: SINAMOS, Dirección General de Organizaciones Culturales y Profesionales.

SAPIENCIA de ZAPATA, S., MACEDA RASSIT, V. \& VIAÑA UZIEDA, J., 1997 - Inventario de la cerámica aymara y quechua, 84 pp.; La Paz: CIMA/Union de Ceramistas Aymaras Quechuas de Bolivia: Centro de Investigación de Energía y Población.

SHEPARD, A. O., 1956 - Ceramics for the Archaeologist, 414 pp.; Washington: Carnegie Institution of Washington.

SHIMADA, I., 1994 - La producción de cerámica en Mórrope, Perú: productividad, especialización y espacio vistos como recursos. In: Tecnología y organización de la producción prehispánica en los Andes (I. Shimada, ed.): 295-319; Lima: Pontificia Universidad Católica del Perú.

SHIMADA, I. (ed.) 1994 - Tecnología y organización de la producción de cerámica prehispánica en los Andes, 517 pp.; Lima: Pontificia Universidad Católica del Perú.

SILLAR, B., 1997 - Reputable Pots and Disreputable Potters: Individual and Community Choice in Present-Day Pottery Production and Exchange in the Andes. In: Not so much a Pot, more a Way of Life (C. G. Cumberpatch \& P. Blinkhorn): 1-20; Oxford: Oxbow Books. 
SILLAR, B., 2000 - Shaping Culture: Making Pots and Constructing Households. An Ethnoarchaeological Study of Pottery Production, Trade and Use in the Andes, viii + 219 pp.; Oxford: BAR.

SILLAR, B. \& RAMÓN JOFFRÉ, G., 2016 - Using the present to interpret the past: The role of ethnographic studies in Andean Archaeology. World Archaeology, 48 (5): 656-673.

SJÖMAN, L., 1989 - Jatunpamba, tierra de alfareras. Cuadernos de cultura popular CIDAP (número especial) 14: 48 pp.

SJÖMAN, L., 1992 - Vasijas de barro: la cerámica popular en el Ecuador, 404 pp.; Cuenca: CIDAP.

SPAHNI, J.-C., 1966 - La cerámica popular del Perú, 131 pp.; Lima: Peruano-Suiza.

STEIN, W., 1961-Hualcan. Life in the Highlands of Peru, 383 pp.; Ithaca: Cornell University Press.

TEJADA, E. de, 1966 - Alfarería de Pujilí. Revista del Folklore Ecuatoriano, 2: 101-118.

TELLO, J. C., 1938 - Arte Antiguo Peruano. Álbum fotográfico de las principales especies arqueológicas de cerámica Muchik existentes en los museos de Lima. Primera parte: tecnología y morfología. Inca. Revista de estudios antropológicos, 2: VII-LXII, 1-280.

TSCHAUNER, H., 2009 - "Los Olleros no son del Inka", Especialización Artesanal y Economía Política en Los Andes: El Caso de los Alfareros de la Pampa de Burros. Revista chilena de Antropología, 20: 261-296.

TSCHOPIK, H. Jr., 1950 - An Andean Ceramic Tradition in Historical Perspective. American Antiquity, 15 (3): 196-218.

VALDEZ, L., 1997 - Ecology and Ceramic Production in an Andean Community: A Reconsideration of the Evidence. Journal of Anthropological Research, 53 (1): 6585.

VARGAS, J. P., 2018 Ms. - Producción alfarera en el Austro ecuatoriano, una perspectiva etnoarqueológica, ponencia presentada en la IX Reunión de Teoría Arqueológica de América del Sur (TAAS), Ibarra (Ecuador), 6 de junio de 2018.

WAGNER, U., GEBHARD, R., MURAD, E., RIEDERER, J., SHIMADA, I. \& WAGNER, F., 1994 - Kiln firing at Batán Grande: today and in Formative times. In: Archaeometry of Pre-Columbian Sites and Artifacts (D. Scott \& P. Meyers, eds.): 67-84; Marina del Rey: Getty Conservation Institute.

WILLIAMS, E., 2019 - Producción de cerámica en la región tarasca de Michoacán, 415 pp.; Zamora: El Colegio de Michoacán.

WYLIE, A., 1985 -The Reaction against Analogy. Advances in Archaeological Method and Theory, 8: 63-111. 